Research Paper

\title{
Hyperhomocysteinemia Associates with Small Vessel Disease More Closely Than Large Vessel Disease
}

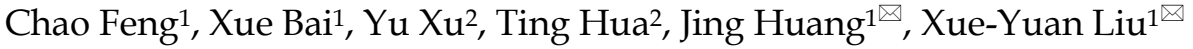 \\ 1. Department of Neurology, Shanghai Tenth People's Hospital, Tongji University, School of Medicine, China; \\ 2. Department of Radiology, Shanghai Tenth People's Hospital, Tongii University, School of Medicine, China.
}

$\triangle$ Corresponding author: Xue-Yuan Liu, MD, Tel: 86-21-66302582, e-mail: xylshtj@yahoo.com.cn; Co-correspondence to Jing Huang, MD, Tel: 86-21-66301167, e-mail: huangjing.neurology@gmail.com. Department of Neurology, Shanghai Tenth People's Hospital of Tongji University, Middle Yanchang Rd. 301\#, Zhabei District, Shanghai, China.

(c) Ivyspring International Publisher. This is an open-access article distributed under the terms of the Creative Commons License (http://creativecommons.org/ licenses/by-nc-nd/3.0/). Reproduction is permitted for personal, noncommercial use, provided that the article is in whole, unmodified, and properly cited.

Received: 2012.09.21; Accepted: 2013.02.14; Published: 2013.02.28

\begin{abstract}
Background: Hyperhomocysteinemia was believed to be an independent risk factor for stroke and associate with small vessel disease (SVD) related stroke and large vessel disease (LVD) related stroke differently. However it's still unclear which type of stroke associated with homocysteine $(\mathrm{HCY})$ more strongly because the conclusions of previous studies were contradictory. In this study we focused on the subclinical angiopathies of stroke, i.e., SVD and LVD instead of stroke subtypes and sought to compare the associations between $\mathrm{HCY}$ level and different angiopathies. Methods: 324 non-stroke patients were enrolled. Sex, age, HCY level and other vascular risk factors were collected. MRI and angiographies were used to determine the type of angiopathies and their severity, i.e., the scores of leukoaraiosis (LA), plaques and numbers of silent brain infarctions (SBI). LVD was defined as the presence of atherosclerotic plaques of cerebral arteries. SVD was defined as the presence of either LA or SBI. 230 patients were deemed to have LVD; 180 patients were deemed to have SVD. Spearman's correlation test and logistic regression were used to analyze the association between HCY level and different angiopathies. Results: The correlation between $\mathrm{HCY}$ level and scores of plaques was weaker than that of the scores of LA and numbers of SBI. Hyperhomocysteinemia was an independent risk factor for SVD $(O R=1.315, P<0.001)$, whereas the association between HCY level and LVD was not that significant $(O R=1.058, P=0.075)$. Conclusion: $\mathrm{HCY}$ level associated with SVD more strongly than LVD.
\end{abstract}

Key words: Hyperhomocysteinemia; small vessel disease; large vessel disease; homocysteine.

\section{Introduction}

Homocysteine (HCY) is a sulfur amino acid which functions as a precursor for L-methionine synthesis and is associated with the metabolism of folic acid and vitamin B B $^{1}$. Elevated HCY level, i.e., hyperhomocysteinemia could result in different angiopathies through the impairment of endothelial function, increased oxidative stress, and disorder of lipid metabolism ${ }^{2,3}$, and is widely regarded as an independent risk factor for stroke ${ }^{4,5}$. There're different studies showing that hyperhomocysteinemia might associate differently with different types of stroke $\mathrm{e}^{5-10}$, which is reasonable because the mechanism of angi- opathy underlying stroke is various, such as hypertensive arteriosclerosis related small vessel disease (SVD) which leads to lacunar stroke ${ }^{11,12}$ and atherosclerosis related large vessel disease (LVD) which usually causes infarctions with large size ${ }^{13}$. However, there's still no conclusion about the heterogeneity of the association between HCY and different types of stroke; some studies reported stronger associations with SVD related infarctions ${ }^{5,7,8}$, while some others demonstrated the association with LVD related infarctions was stronger $6,9,10$.

There're different explanations for this discrep- 
ancy. The race's heterogeneity involved in the studies might be a confounding factor. Another explanation might be that these studies focused on stroke subtypes but not angiopathies themselves and might result in bias because the subtype of stroke was determined by infarction sizes and risk factors combined with vascular assessment and other clinical information, and could not reflect the underlying angiopathies truthfully especially for those patients with both SVD and LVD ${ }^{14,15}$ who might account for a large part of the subjects enrolled in these studies.

LVD is well visualized on images of angiographies where atherosclerosis and stenosis of main arteries could be shown directly, whereas SVD could only be judged according to the indirect image features such as leukoaraiosis (LA) and silent brain infarctions (SBI) ${ }^{16}$. In this study, we focused on the subclinical status of stroke, i.e., angiopathies including SVD and LVD. Based on the evaluation of LA, SBI and atherosclerosis of extra- and intra-cerebral arteries identified by angiographies, we tried to explore the associations between HCY and different types of angiopathy.

\section{Subjects and Methods}

\section{General information}

This study was a cross-sectional study based on the patients of $10^{\text {th }}$ People's Hospital in Shanghai from September 2011 to August 2012. Subjects attended to the department of Neurology mainly with non-specific symptoms such as headache, dizziness, or with the fear of stroke because the presence of risk factors, and were consecutively enrolled according to the following criteria: 1) age from 40-85 years; 2) no history of stroke, myocardial infarction, or confirmed diagnosis of angina and peripheral vascular disease or correlated symptoms, no acute stroke identified in MR images; 3) no claustrophobia and metallic implants. Subjects were evaluated with the following protocol: brain magnetic resonance imaging (MRI), electrocardiogram, vascular assessment of both intraand extra-cerebral arteries involving either computed tomography angiography (CTA) or magnetic resonance angiography (MRA), as well as blood tests for blood glucose, lipid, and HCY. Leukoaraiosis, silent brain infarctions and atherosclerosis were evaluated respectively according to MRI and angiography. Information about age, gender, body mass index (BMI), history of smoking and drinking, presence of hypertension of each subject was also collected. All procedures were carried out after the consent of subjects and proved by the Institutional Review Board of our hospital.

\section{MR protocol}

The MR images obtained in this study were mostly based on a $1.5 \mathrm{~T}$ scanner (Philips, Netherlands) and partly based on a $3.0 \mathrm{~T}$ scanner (Siemens, Germany). The MRI protocol consisted of a T1-weighted image [repetition time/echo time $(\mathrm{TR} / \mathrm{TE})=101 / 1.92$ for $1.5 \mathrm{~T}, 2000 / 9$ for $3.0 \mathrm{~T}$ scanner], fluid attenuated inversion recovery images (FLAIR) $(\mathrm{TR} / \mathrm{TE}=6000 / 110$ for $1.5 \mathrm{~T}, 8500 / 94$ for $3.0 \mathrm{~T}$ scanner), and DWI (TR/TE $=3393 / 86$ for $1.5 \mathrm{~T}, 6000 / 94$ for $3.0 \mathrm{~T}$ scanner) in the axial plane, as well as a T2-weighted image (TR/TE $=1940 / 120$ for $1.5 \mathrm{~T}$, $4540 / 96$ for $3.0 \mathrm{~T}$ scanner) in the sagittal plane with 16 layers.

\section{Image review and criteria}

All the images were reviewed by 2 experienced readers blind to the clinical information. The discrepancy of the presence and severity of LA, SBI and LVD was resolved by a visual consensus. SVD was defined as the presence of either LA or SBI ${ }^{12,16}$. LA was defined as focal or confluent hyperintensities in the deep or periventricular area on FLAIR images ${ }^{17}$, and was scored according to Fazekas' grading scale ${ }^{18}$. Periventricular hyperintensities of only a pencil-thin lining and caps with thickness less than $3 \mathrm{~mm}$ were excluded from the analysis because their visualization might be influenced by other confounding factors. SBI was defined as a small deep focal hypo-intensity on T1-weighted image and corresponding hyper-intensity on T2-weighted image and hypo-intense with a hyper-intense boundary on FLAIR images, 3-15 $\mathrm{mm}$ in size, with no corresponding stroke ${ }^{19}$. LVD was defined the presence of atherosclerotic plaques of extra- and intra-cerebral main arteries including carotid artery, middle cerebral artery, anterior cerebral artery, posterior cerebral artery, vertebral and basilar arteries shown on CTA or MRA. Plaque score was rated as grade 0 for no plaque, 1 for one small plaque with stenosis $<30 \%, 2$ for one medium plaque with stenosis $30-49 \%$ or multiple small plaques, 3 for one large plaque with stenosis $50-75 \%$ or multiple plaques with stenosis $30-49 \%$, 4 for stenosis of $76-100 \%{ }^{20}$.

\section{Data Analysis}

All data were analysed with SPSS 18.0. Categorical data and measurement data were analyzed by chi-square test and Student's $t$ test respectively. Correlations between scores of LA/numbers of SBI/scores of plaques and HCY level were examined with Spearman's correlation test. Logistic regression models were constructed to determine the risk factors for the presence of SVD/LVD, with age, gender, BMI, smoking, drinking, HCY level, presence of hypertension, diabetes, dyslipedemia and presence of 
LVD/SVD added into the models. A value of $P<0.05$ was considered statistically significant.

\section{Results}

324 patients $(66.9 \pm 10.7$ years old, 165 men and 159 women) were enrolled into this study. Characteristics of patients with or without LVD, and patients with or without SVD were listed in Table 1 and Table 2 respectively. Patients with LVD were significantly older, with significantly higher blood pressure, blood glucose, blood lipid, and higher HCY level than those without LVD. Patients with SVD were significantly older too, with significantly higher blood pressure and significantly higher HCY level than those without SVD. There were significant correlations between HCY level and either scores of LA $(r=0.379, \mathrm{P}<0.001)$ or numbers of SBI $(r=0.339, \mathrm{P}<0.001)$ according to the results of Spearman's correlation test. A weaker correlation was also observed between HCY level and scores of plaques $(\mathrm{r}=0.131, \mathrm{P}=0.018)$. Binary logistic regressions showed that hypertension, diabetes and dyslipedemia were independent risk factors for LVD, and the association between HCY level and LVD was not that significant $(\mathrm{OR}=1.058, \mathrm{P}=0.075)$; while hyperhomocysteinemia was independent risk factor for SVD $(\mathrm{OR}=1.315, \mathrm{P}<0.001)$. Details of logistic regressions were shown in Table 3 and Table 4.

Table I. Characteristics of patients with or without LVD.

\begin{tabular}{llll}
\hline & $\begin{array}{l}\text { Patients without } \\
\text { LVD (n=94) }\end{array}$ & PVD (n=230) & \\
\hline Age, years (SD) & $59.9(8.7)$ & $69.7(10.1)$ & $<0.001$ \\
Female, \% (n) & $53.2(50)$ & $47.4(109)$ & 0.392 \\
Hypertension, \% (n) & $31.9(30)$ & $60.0(138)$ & $<0.001$ \\
Diabetes Mellitus, \% (n) & $5.3(5)$ & $23.5(54)$ & $<0.001$ \\
Dyslipedemia, \% (n) & $36.2(34)$ & $64.8(149)$ & $<0.001$ \\
BMI (SD) & $24.5(2.6)$ & $25.4(3.0)$ & 0.007 \\
Smoking, \% (n) & $6.4(6)$ & $21.7(50)$ & 0.001 \\
Drinking, \% (n) & $14.9(14)$ & $17.4(40)$ & 0.626 \\
HCY level, umol/1 (SD) & $14.6(4.9)$ & $16.2(6.3)$ & 0.024 \\
\hline
\end{tabular}

Table 2. Characteristics of patients with or without SVD.

\begin{tabular}{llll}
\hline & $\begin{array}{l}\text { Patients } \\
\text { without SVD } \\
(\mathrm{n}=144)\end{array}$ & $\begin{array}{l}\text { Patients } \\
\text { with SVD } \\
(\mathrm{n}=180)\end{array}$ & $\mathrm{P}$ \\
\hline Age, years (SD) & $58.7(6.9)$ & $73.4(8.4)$ & $<0.001$ \\
Female, \% (n) & $47.9(69)$ & $50.0(90)$ & 0.738 \\
Hypertension, \% (n) & $28.5(41)$ & $70.6(127)$ & $<0.001$ \\
Diabetes Mellitus, \% (n) & $14.6(21)$ & $21.1(38)$ & 0.148 \\
Dyslipedemia, \% (n) & $56.3(81)$ & $56.7(102)$ & $>0.999$ \\
BMI (SD) & $25.1(2.9)$ & $25.2(2.9)$ & 0.653 \\
Smoking, \% (n) & $11.8(17)$ & $21.7(39)$ & 0.026 \\
Drinking, \% (n) & $17.4(25)$ & $16.1(29)$ & 0.767 \\
HCY level, umol/1 (SD) & $13.5(4.0)$ & $17.5(6.6)$ & $<0.001$ \\
\hline
\end{tabular}

Table 3. Logistic regression for predicting LVD.

\begin{tabular}{llll}
\hline & OR & $95 \%$ CI & P \\
\hline Age (years) & 1.138 & $1.087-1.190$ & $<0.001$ \\
Sex & 0.645 & $0.334-1.246$ & 0.191 \\
Hypertension & 2.193 & $1.108-4.339$ & 0.024 \\
Diabetes Mellitus & 11.496 & $3.677-35.940$ & $<0.001$ \\
Dyslipidemia & 5.823 & $2.894-11.717$ & $<0.001$ \\
Smoking & 3.715 & $1.192-11.577$ & 0.024 \\
Drinking & 0.933 & $0.388-2.245$ & 0.878 \\
BMI & 1.075 & $0.958-1.205$ & 0.218 \\
HCY level (umol/1) & 1.058 & $0.994-1.126$ & 0.075 \\
SVD & 0.592 & $0.237-1.478$ & 0.261 \\
\hline
\end{tabular}

Table 4. Logistic regression for predicting SVD.

\begin{tabular}{llll}
\hline & OR & $95 \%$ CI & $\mathrm{P}$ \\
\hline Age (years) & 1.338 & $1.248-1.434$ & $<0.001$ \\
Sex & 0.626 & $0.273-1.435$ & 0.269 \\
Hypertension & 4.935 & $2.143-11.364$ & $<0.001$ \\
Diabetes Mellitus & 1.795 & $0.583-5.524$ & 0.308 \\
Dyslipidemia & 1.012 & $0.397-2.583$ & 0.980 \\
Smoking & 2.513 & $0.786-8.034$ & 0.120 \\
Drinking & 0.597 & $0.192-1.855$ & 0.373 \\
BMI & 1.079 & $0.925-1.258$ & 0.335 \\
HCY level (umol/l) & 1.315 & $1.193-1.450$ & $<0.001$ \\
LVD & 0.522 & 0.1881 .447 & 0.211 \\
\hline
\end{tabular}

\section{Discussion}

This study showed that, HCY level was higher in patients with LVD or SVD; although positive correlations were found between HCY level and either of the scores of LA, numbers of SBI, and scores of plaques, the correlation between HCY level and scores of plaques was weaker; HCY level was an independent risk factor for SVD, whereas after adjusting for other confounding factors, the association between $\mathrm{HCY}$ level and LVD was less significant.

This was the first study that compared the strength of associations between HCY level and two main subclinical angiopathies of stroke. The strength of this study mainly involved the relatively large amount of subjects in subclinical status of stroke instead of different types of stroke, the score systems for different types of angiopathies and various statistical methods for the comparison. However, some limitations were inevitable. The non-prospective study design and hospital based study-population restricted the significance of our results. Besides, although we tried to exclude the atherosclerosis related disease of other systems through the restriction of including criteria, the angiopathies of cerebral arteries still could not represent the status of entire vascular system 
truthfully. Furthermore, the presence and types of angiopathy were determined by imaging tools without pathological study, thus some early stages of the angiopathies and atherosclerosis of small vessels that could not be shown by images might be neglected.

Hyperhomocysteinemia was identified as an independent risk factor for vascular disease including stroke at the end of last century ${ }^{21}$. Some subsequent studies explored the associations between HCY level and different stroke subtypes and mainly concluded that LVD and SVD related infarctions were two main types of stroke that associated with hyperhomocysteinemia ${ }^{5-8}$. It was reasonable because they were both caused by angiopathies associated with endothelia dysfunction ${ }^{3,22-25}$ which might be the main pathologic effect of hyperhomocysteinemia to vessels proved by experimental work $^{2,3}$, and some clinical studies already proved that HCY level was associated with the covert phenotypes of SVD such as LA and SBI26-28. However, LVD and SVD represented different angiopathies, i.e., atherosclerosis and lipohyalinosis or fibrinoid necrosis related arteriosclerosis respectively ${ }^{12}$. And the specific mechanism of endothelia dysfunction and the specific role of endothelia dysfunction in the pathologic mechanism of angiopathies might be different to some extent. So it's reasonable that they might have different vascular risk factor profiles. Previous studies already proved that hypertension was the main risk factor for SVD, while diabetes and dyslipedemia were more important to LVD $^{29}$. This study confirmed that hyperhomocysteinemia was an independent risk factor for SVD too, while its association with LVD was not that strong. Our result was not in accordance with some studies about HCY and stroke subtypes6, 9, 10. But this discrepancy was reasonable because this study focused on angiopathies instead of stroke subtypes. Besides, we noticed that few studies about the associations between HCY level and stroke subtypes had taken LA and SBI into account as confounding factors. Considering the high prevalence of LA and SBI especially in the patients of stroke ${ }^{17,30}$, and their strong association with HCY level ${ }^{26-28}$, some bias of the results was inevitable.

However, we are not here to deny the correlation between HCY level and LVD; on the contrary, we did notice a positive correlation between HCY level and plaque scores, even though it was weaker than the others. On the other hand, although we confirmed a strong association between HCY level and SVD, the role of HCY in the mechanism of angiopathies is still not as clear as other risk factors such as hypertension. There were already several studies about HCY-lowering therapy in the treatment of stroke and coronary artery disease; some of them suggested a positive result ${ }^{31,32}$. And considering the positive correlation between HCY and different subclinical angiopathies especially SVD, HCY might become another potential target for the secondary prevention of stroke and other vascular disease too. In the future, more experimental and clinical studies are required to clarify the specific role of HCY in different angiopathies and to verify the effect of HCY related therapy.

\section{Acknowledgement}

This study was supported by grants from the National Natural Science Foundation of China (No. 81000492, No. 30971029, No. 81171163).

\section{Conflict of interest}

None.

\section{References}

1. Sen U, Mishra PK, Tyagi N, Tyagi SC. Homocysteine to hydrogen sulfide or hypertension. Cell Biochem Biophys 2010;57:49-58

2. Weiss N. Mechanisms of increased vascular oxidant stress in hyperhomocys-teinemia and its impact on endothelial function. Curr Drug Metab 2005;6:27-36.

3. Abahji TN, Nill L, Ide N, Keller C, Hoffmann U, Weiss N. Acute Hyperhomocysteinemia Induces Microvascular and Macrovascular Endothelial Dysfunction. Archives of Medical Research 2007;38:411-416.

4. Wierzbicki AS. Homocysteine and cardiovascular disease: a review of the evidence. Diabetes \& vascular disease research: official journal of the International Society of Diabetes and Vascular Disease 2007;:143.

5. Iso H. Serum Total Homocysteine Concentrations and Risk of Stroke and Its Subtypes in Japanese. Circulation 2004;109:2766-2772.

6. Eikelboom JW, Hankey GJ, Anand SS, Lofthouse E, Staples N, Baker RI. Association Between High Homocyst(e)ine and Ischemic Stroke due to Large- and Small-Artery Disease but Not Other Etiologic Subtypes of Ischemic Stroke. Stroke 2000;31:1069-1075.

7. Perini F, Galloni E, Bolgan I, et al. Elevated plasma homocysteine in acute stroke was not associated with severity and outcome: stronger association with small artery disease. Neurological Sciences 2005;26:310-318.

8. Khan U, Crossley C, Kalra L, et al. Homocysteine and Its Relationship to Stroke Subtypes in a UK Black Population: The South London Ethnicity and Stroke Study. Stroke 2008;39:2943-2949.

9. Tan NCK. Hyperhomocyst(e)inemia and Risk of Ischemic Stroke Among Young Asian Adults. Stroke 2002;33:1956-1962.

10. Parnetti L, Caso V, Santucci A, et al. Mild hyperhomocysteinemia is a risk-factor in all etiological subtypes of stroke. Neurological Sciences 2004;25:13-17.

11. Lee SH, Bae HJ, Yoon BW, Kim H, Kim DE, Roh JK. Low Concentration of Serum Total Cholesterol Is Associated With Multifocal Signal Loss Lesions on Gradient-Echo Magnetic Resonance Imaging: Analysis of Risk Factors for Multifocal Signal Loss Lesions. Stroke 2002;33:2845-2849.

12. Pantoni L. Cerebral small vessel disease: from pathogenesis and clinical characteristics to therapeutic challenges. Lancet Neurol 2010;9:689-701.

13. Murat Sumer M, Erturk O. Ischemic stroke subtypes: risk factors, functional outcome and recurrence. Neurol Sci 2002;22:449-454.

14. Kloppenborg RP, Nederkoorn PJ, van der Graaf Y, Geerlings MI. Homocysteine and cerebral small vessel disease in patients with symptomatic atherosclerotic disease. The SMART-MR study. Atherosclerosis 2011;216:461-466.

15. Jung KW, Shon Y-M, Yang DW, Kim BS, Cho AH. Coexisting Carotid Atherosclerosis in Patients with Intracranial Small- or Large-Vessel Disease. Journal of Clinical Neurology 2012;8:104.

16. Moran C, Phan TG, Srikanth VK. Cerebral small vessel disease: a review of clinical, radiological, and histopathological phenotypes. Int J Stroke 2012;7:36-46.

17. Xiong YY, Mok V. Age-related white matter changes. J Aging Res 2011;2011:617927.

18. Franz Fazekas JBC, Abass Alavi, Howard I. Hurtig, Robert A. Zimmerman. MR signal abnormalities at $1.5 \mathrm{~T}$ in Alzheimer's dementia and normal aging. AJR 1987;149:351-356. 
19. Zhu YC, Dufouil C, Tzourio C, Chabriat H. Silent brain infarcts: a review of MRI diagnostic criteria. Stroke 2011;42:1140-1145.

20. Lien L-M, Hsieh Y-C, Bai C-H, et al. Association of blood active matrix metalloproteinase-3 with carotid plaque score from a community population in Taiwan. Atherosclerosis 2010;212:595-600.

21. R. Clarke LD, K. Robinson, E. Naughten, S. Cahalane, B. Fowler, I. Garham. Hyperhomocysteinemia: An Independent Risk Factor for Vascular Disease. NEJM 1991; 324:1149-1155.

22. Knottnerus ILH, Ten Cate H, Lodder J, Kessels F, van Oostenbrugge RJ. Endothelial Dysfunction in Lacunar Stroke: A Systematic Review. Cerebrovascular Diseases 2009;27:519-526.

23. Landmesser U. Endothelial Function: A Critical Determinant in Atherosclerosis? Circulation 2004;109:27-33

24. Markus HS. Genes, endothelial function and cerebral small vessel disease in man. Experimental Physiology 2007;93:121-127.

25. Stevenson SF, Doubal FN, Shuler K, Wardlaw JM. A Systematic Review of Dynamic Cerebral and Peripheral Endothelial Function in Lacunar Stroke Versus Controls. Stroke 2010:41:e434-e442.

26. Öncel Ç, Demir S, Güler S, Cenikli U, Tabak E, Kıroğlu Y. Association between cholesterols, homocysteine and silent brain infarcts. Internal Medicine Journal 2009;39:150-155.

27. Tseng Y-L, Chang Y-Y, Liu J-S, Su C-S, Lai S-L, Lan M-Y. Association of plasma homocysteine concentration with cerebral white matter hyperintensity on magnetic resonance images in stroke patients. Journal of the Neurological Sciences 2009;284:36-39.

28. Censori B, Partziguian T, Manara O, Poloni M. Plasma homocysteine and severe white matter disease. Neurological Sciences 2007;28:259-263.

29. Khan U, Porteous L, Hassan A, Markus HS. Risk factor profile of cerebral small vessel disease and its subtypes. J Neurol Neurosurg Psychiatry 2007;78:702-706.

30. Vermeer SE, Longstreth WT, Jr., Koudstaal PJ. Silent brain infarcts: a systematic review. Lancet Neurol 2007;6:611-619.

31. Potter K, Hankey GJ, Green DJ, Eikelboom J, Jamrozik K, Arnolda LF. The effect of long-term homocysteine-lowering on carotid intima-media thickness and flow-mediated vasodilation in stroke patients: a randomized controlled trial and meta-analysis. BMC Cardiovascular Disorders 2008;8:24.

32. Mager A, Orvin $\mathrm{K}$, Koren-Morag $\mathrm{N}$, et al. Impact of homocysteine-lowering vitamin therapy on long-term outcome of patients with coronary artery disease. Am J Cardiol 2009;104:745-749. 